\title{
Dividend Problems with a Barrier Strategy in the Dual Risk Model until Bankruptcy
}

\author{
Donghai Liu ${ }^{1}$ and Zaiming Liu ${ }^{2}$ \\ ${ }^{1}$ Department of Mathematics, Hunan University of Science and Technology, Xiangtan, Hunan 411201, China \\ ${ }^{2}$ Department of Mathematics and Statistics, Central South University, Changsha, Hunan 410075, China
}

Correspondence should be addressed to Donghai Liu; dhliu@hnust.edu.cn

Received 29 March 2014; Revised 19 July 2014; Accepted 21 July 2014; Published 11 August 2014

Academic Editor: Daqing Jiang

Copyright ( 2014 D. Liu and Z. Liu. This is an open access article distributed under the Creative Commons Attribution License, which permits unrestricted use, distribution, and reproduction in any medium, provided the original work is properly cited.

\begin{abstract}
The paper studies the dual risk model with a barrier strategy under the concept of bankruptcy, in which one has a positive probability to continue business despite temporary negative surplus. Integrodifferential equations for the expectation of the discounted dividend payments and the probability of bankruptcy are derived. Moreover, when the gain size distribution is exponential, explicit solutions for the expected dividend payments and the bankruptcy probability are obtained for constant bankruptcy rate function. It also provided some numerical examples to illustrate the applications of the explicit solutions.
\end{abstract}

\section{Introduction}

In the continuous time dual risk model, the company's surplus process $\{U(t), t \geq 0\}$ with initial surplus $U(0)=u$ is given by

$$
U(t)=u-c t+S(t)=u-c t+\sum_{i=1}^{N(t)} X_{i}, \quad t \geq 0,
$$

where $c>0$ is the constant rate of expenses per unit time, $\{N(t), t \geq 0\}$ is a Poisson process with intensity $\lambda>0$, and the gain size $\left\{X_{i}, i \geq 1\right\}$ is a sequence of independent and identically distributed positive continuous random variables with finite mean, independent of $\{N(t), t \geq 0\}$. Assume all $X_{i}$ have the same distribution as a generic random variable $X$, which has probability density function $f(x)$ and cumulative distribution function $F(x)$. Under model (1), the expected increase in surplus per unit time is $\mu=\lambda E X-c$, and it is assumed that $\mu>0$.

Since De Finetti [1] proposed dividend strategies for an insurance risk model, the risk model in the presence of dividend payments has become a more and more popular topic in risk theory. In recent years, quite a few interesting papers have been written on the dual risk model with dividend strategy. Avanzi et al. [2] studied the expected total discounted dividends in the dual risk model with barrier strategy. Avanzi and Gerber [3] studied the optimal dividends in the dual risk model with diffusion. $\mathrm{Ng}[4]$ studied the expected discounted dividend in dual risk model with threshold dividend strategy. Dai et al. [5] studied the optimal dividend strategies in the dual risk model with capital injections.

Albrecher and Lautscham [6] made a distinction between ruin and bankruptcy, in the traditional actuarial model; if the surplus is negative, the company is ruined and has to go out of business; in particular, no dividends are paid after ruin. They consider a relaxation of the ruin concept to the concept of bankruptcy, in which the company with a negative surplus is assumed to be able to continue doing business as usual until bankruptcy takes place, and bankruptcy means that the company goes out of business. Concretely, a suitable bankruptcy rate function $\omega(u)$ depending on the size of the negative surplus, which is defined on $-a \leq u<0$, zero for $u>0$, and $\omega(u)=\infty$ for $u<-a$. This is a nonincreasing function; whenever the negative surplus is $u, \omega(u) d t$ is the probability of bankruptcy within dt time units. Albrecher and Lautscham [6] considered the optimal dividend barrier 
in diffusion risk model until bankruptcy. In this paper, we extend the idea of ruin to more general bankruptcy concept in dual risk model.

Let us now consider some of the basic definition and notation for the risk model. Let $\tau$ be the bankruptcy time of the surplus process with dividend payments and define the overall probability of bankruptcy as

$$
\psi(u, b)=P\{\tau<\infty \mid U(0)=u\} .
$$

A barrier dividend strategy is given by a parameter $b \geq 0$; if at a potential dividend payment time, the surplus is above $b$; the excess is paid as a dividend and then the aggregate dividends $D(t)$ till time $t$ is

$$
D(t)=\left(U\left(t^{-}\right)-b\right)_{+},
$$

where $\left(U\left(t^{-}\right)-b\right)_{+}=\max \left\{U\left(t^{-}\right)-b, 0\right\}$.

The dividends are discounted at a constant force of interest $\delta \geq 0$, the total discounted dividends until bankruptcy are

$$
D_{u, b}=\int_{0}^{\tau} e^{-\delta t} d D(t),
$$

and the expected discounted value of dividends by $V(u, b)=$ $E\left[D_{u, b}\right]$.

The purpose of this paper is to present the expected value of the discounted sum of all dividend payments until bankruptcy and the probability of bankruptcy in the dual risk model. In Section 2, integrodifferential equations for the expected dividend payments until bankruptcy are derived; moreover, explicit solutions are also obtained under constant bankruptcy rate function and exponential gain size; finally, we provide some numerical examples with illustrations for the expected dividend payments under the concept of bankruptcy. In Section 3, we derive equations for the probability of bankruptcy $\psi(u, b)$, which are solved explicitly for constant bankrupt rate function and exponential gain size and we also provide numerical examples with illustrations for the probability of bankruptcy.

\section{The Expectation of the Discounted Dividend Payments}

In this section, we derive integrodifferential equations for $V(u, b)$; the results are summarized in the following theorem. At first, we define

$$
V(u, b)= \begin{cases}V_{1}(u, b), & u<0 \\ V_{2}(u, b), & 0 \leq u<b \\ u-b+V_{2}(b, b), & u \geq b\end{cases}
$$

Similarly, we can define $\psi_{i}(u, b)$ in next section as $V_{i}(u, b)(i=$ $1,2)$.

\subsection{Integrodifferential Equations for $V(u, b)$}

Theorem 1. $V_{1}(u, b)$ and $V_{2}(u, b)$ satisfy the following system of integrodifferential equations:

$$
\begin{aligned}
& c V_{1}^{\prime}(u, b)+(\lambda+\delta+\omega(u)) V_{1}(u, b) \\
& -\lambda \int_{u}^{0} V_{1}(x, b) f_{X}(x-u) d x-\lambda \int_{0}^{b} V_{2}(x, b) \\
& \cdot f_{X}(x-u) d x-\lambda V_{2}(b, b) \bar{F}(b-u) \\
& \quad-\lambda \int_{b-u}^{\infty}\left(1-F_{X}(x)\right) d x=0, \quad u<0 \\
& c V_{2}^{\prime}(u, b)+(\lambda+\delta) V_{2}(u, b) \\
& \quad-\lambda \int_{u}^{b} V_{2}(x, b) f_{X}(x-u) d x-\lambda \int_{b-u}^{\infty}\left(1-F_{X}(x)\right) d x \\
& -\lambda V_{2}(b, b) \bar{F}(b-u)=0, \quad 0 \leq u<b .
\end{aligned}
$$

In addition, $V_{1}(u, b)$ and $V_{2}(u, b)$ satisfy

$$
\begin{gathered}
V_{1}\left(0^{-}, b\right)=V_{2}\left(0^{+}, b\right), \\
\lim _{u \rightarrow-\infty} V_{1}(u, b)=0, \\
V_{2}^{\prime}\left(0^{+}, b\right)-V_{1}^{\prime}\left(0^{-}, b\right)=\frac{\omega\left(0^{-}\right)}{c} V_{1}\left(0^{-}, b\right) .
\end{gathered}
$$

Proof. When $u>b$, the surplus drops to level $b$ immediately due to the initial payment of dividends and thus

$$
V_{2}(u, b)=u-b+V_{2}(b, b)
$$

when $u<0$, conditioning on the first occurrence time of either a gain or an event of bankruptcy up to time $t$ yields that

$$
\begin{aligned}
V_{1}(u, b)= & (1-\lambda t)(1-\omega(u) t) e^{-\delta t} V_{1}(u-c t, b) \\
& +(1-\lambda t) \omega(u) t \cdot 0+(1-\omega(u) t) \lambda t \\
& \cdot\left[\int_{0}^{-u+c t} V_{1}(u-c t+x, b) f_{X}(x) d x\right. \\
& +\int_{-u+c t}^{b-u+c t} V_{2}(u-c t+x, b) f_{X}(x) d x \\
& \left.+\int_{b-u+c t}^{\infty}\left(u-c t+x-b+V_{2}(b, b)\right) f_{X}(x) d x\right] \\
& +o(t)=0 .
\end{aligned}
$$


We differentiate (12) with respect to $t$, and by taking the $\operatorname{limit} t \rightarrow 0$ we can get

$$
\begin{aligned}
& c V_{1}^{\prime}(u, b)+(\lambda+\delta+\omega(u)) V_{1}(u, b) \\
& -\lambda \int_{0}^{-u} V_{1}(u+x, b) f_{X}(x) d x \\
& -\lambda \int_{-u}^{b-u} V_{2}(u+x, b) \\
& \cdot f_{X}(x) d x-\lambda \int_{b-u}^{\infty}(u+x-b) f_{X}(x) d x \\
& -\lambda V_{2}(b, b) \bar{F}(b-u)=0 .
\end{aligned}
$$

That is,

$$
\begin{aligned}
& c V_{1}^{\prime}(u, b)+(\lambda+\delta+\omega(u)) V_{1}(u, b) \\
& -\lambda \int_{u}^{0} V_{1}(x, b) f_{X}(x-u) d x-\lambda \int_{0}^{b} V_{2}(x, b) \\
& \cdot f_{X}(x-u) d x-\lambda \int_{b-u}^{\infty}\left(1-F_{X}(x)\right) d x \\
& -\lambda V_{2}(b, b) \bar{F}(b-u)=0 .
\end{aligned}
$$

When $0 \leq u<b$,

$$
\begin{aligned}
V_{2}(u, b)= & (1-\lambda t) e^{-\delta t} V_{2}(u-c t, b) \\
& +\lambda t \int_{0}^{b-u+c t} V_{2}(u-c t+x, \mathrm{~b}) f_{X}(x) d x \\
& +\lambda t \int_{b-u+c t}^{\infty}\left(u-c t+x-b+V_{2}(b, b)\right) f_{X}(x) d x \\
& +o(t)=0 .
\end{aligned}
$$
(7).

Using a similar method of deriving (6), we can also obtain

Letting $u \downarrow 0$ in (12) and $u \uparrow 0$ in (15), it follows that $V(u, b)$ is continuous at $u=0$ as long as $\omega(0)$ is bounded; that is,

$$
V_{1}\left(0^{-}, b\right)=V_{2}\left(0^{+}, b\right)
$$

the continuity of $V(u, b)$ at $u=0$; we can deduce from (6) and (7) for $u \rightarrow 0$ that

$$
V_{2}^{\prime}\left(0^{+}, b\right)-V_{1}^{\prime}\left(0^{-}, b\right)=\frac{\omega\left(0^{-}\right)}{c} V_{1}\left(0^{-}, b\right) ;
$$

we know that if $u \rightarrow-\infty$, the bankruptcy takes place, so $\lim _{u \rightarrow-\infty} V_{1}(u, b)=0$ is obvious.

2.2. Explicit Expressions for $V(u, b)$. We assume in this subsection that $\omega(u)$ is constant; the positive constant is denoted as $\omega$; that is,

$$
\omega(u)=\omega, \quad-a \leq u<0 .
$$

For simplicity, we will assume throughout the rest of the paper that the gain size is exponentially distributed $F(x)=$ $1-e^{-\alpha x}$.

In this case, (6) can be rewritten as

$$
\begin{aligned}
& c V_{1}^{\prime}(u, b)+(\lambda+\delta+\omega) V_{1}(u, b) \\
& -\lambda \int_{u}^{0} V_{1}(x, b) \alpha e^{-\alpha(x-u)} d x \\
& -\lambda \int_{0}^{b} V_{2}(x, b) \alpha e^{-\alpha(x-u)} d x \\
& -\lambda V_{2}(b, b) e^{-\alpha(b-u)}-\lambda \int_{b-u}^{\infty} e^{-\alpha x} d x=0 .
\end{aligned}
$$

That is,

$$
\begin{aligned}
& c V_{1}^{\prime}(u, b)+(\lambda+\delta+\omega) V_{1}(u, b) \\
& -\lambda \int_{u}^{0} V_{1}(x, b) \alpha e^{-\alpha x} e^{\alpha u} d x-\lambda \int_{0}^{b} V_{2}(x, b) \alpha e^{-\alpha x} \\
& \cdot e^{\alpha u} d x-\lambda V_{2}(b, b) e^{-\alpha(b-u)}-\frac{\lambda}{\alpha} e^{-\alpha(b-u)}=0 .
\end{aligned}
$$

Applying the operator $(d / d u-\alpha)$ to $(20)$, we obtain the differential equation

$$
\begin{gathered}
c V_{1}^{\prime \prime}(u, b)+(\lambda+\delta+\omega-c \alpha) V_{1}^{\prime}(u, b) \\
-\alpha(\delta+\omega) V_{1}(u, b)=0
\end{gathered}
$$

Hence the solution of (21) is of the form

$$
V_{1}(u, b)=A_{1} e^{-r_{1} u}+B_{1} e^{s_{1} u}
$$

where $A_{1}, B_{1}$ are arbitrary coefficients and $-r_{1}<0$ and $s_{1}>0$ are the two solutions to the characteristic equation about $\eta$ :

$$
c \eta^{2}+(\lambda+\delta+\omega-c \alpha) \eta-\delta(\alpha+\omega)=0
$$

From $\lim _{u \rightarrow-\infty} V_{1}(u, b)=0$, it follows that $A_{1}=0$.

Analogously, we rewrite (7) as

$$
\begin{aligned}
& c V_{2}^{\prime}(u, b)+(\lambda+\delta) V_{2}(u, b) \\
& \quad-\lambda \int_{u}^{b} V_{2}(x, b) \alpha e^{-\alpha(x-u)} d x-\frac{\lambda}{\alpha} e^{-\alpha(b-u)} \\
& \quad-\lambda V_{2}(b, b) e^{-\alpha(b-u)}=0 .
\end{aligned}
$$

That is,

$$
c V_{2}^{\prime}(u, b)+(\lambda+\delta-c \alpha) V_{2}^{\prime}(u, b)-\alpha \delta V_{2}(u, b)=0 .
$$

The solution of (25) is of the form

$$
V_{2}(u, b)=A_{2} e^{r_{2} u}+B_{2} e^{s_{2} u},
$$

where $A_{2}, B_{2}$ are constants and $r_{2}, s_{2}$ are the solutions of the equation about $\eta^{\prime}$

$$
c\left(\eta^{\prime}\right)^{2}+(\lambda+\delta-c \alpha) \eta^{\prime}-\delta \alpha=0 .
$$


From (8), we obtain that

$$
B_{1}=A_{2}+B_{2} \text {. }
$$

The condition (10) gives the equation

$$
A_{2} r_{2}+B_{2} s_{2}-B_{1} s_{1}=\frac{\omega}{c} B_{1}
$$

Substituting (26) into (7), we have

$$
A_{2}\left(c r_{2}+\delta\right) e^{r_{2} b}+B_{2}\left(c s_{2}+\delta\right) e^{s_{2} b}=\frac{\lambda}{\alpha} .
$$

Therefore, we have a system of linear equations (28)-(30) for the constants $A_{2}, B_{1}$, and $B_{2}$. Solving the system of linear equations, we have

$$
\begin{aligned}
A_{2}= & \frac{\lambda}{\alpha}\left(s_{2}-\frac{\omega}{c}-s_{1}\right) \\
& \times\left(\left(c r_{2}+\delta\right)\left(s_{2}-\frac{\omega}{c}-s_{1}\right) e^{r_{2} b}\right. \\
& \left.-\left(c s_{2}+\delta\right)\left(r_{2}-\frac{\omega}{c}-s_{1}\right) e^{s_{2} b}\right)^{-1}, \\
B_{1}=\frac{\lambda}{\alpha}\left(s_{2}-r_{2}\right) & \quad\left(\left(c r_{2}+\delta\right)\left(s_{2}-\frac{\omega}{c}-s_{1}\right) e^{r_{2} b}\right. \\
& \left.\quad-\left(c s_{2}+\delta\right)\left(r_{2}-\frac{\omega}{c}-s_{1}\right) e^{s_{2} b}\right)^{-1}, \\
B_{2}=\frac{\lambda}{\alpha} & \left(r_{2}-\frac{\omega}{c}-s_{1}\right) \\
& \times\left(\left(c s_{2}+\delta\right)\left(r_{2}-\frac{\omega}{c}-s_{1}\right) e^{s_{2} b}\right. \\
& \left.\quad-\left(c r_{2}+\delta\right)\left(s_{2}-\frac{\omega}{c}-s_{1}\right) e^{r_{2} b}\right)^{-1} .
\end{aligned}
$$

So we can obtain

$$
\begin{aligned}
V_{1}(u, b)=\frac{\lambda}{\alpha}( & \left(s_{2}-r_{2}\right) \\
& \times\left(\left(c r_{2}+\delta\right)\left(s_{2}-\frac{\omega}{c}-s_{1}\right) e^{r_{2} b}\right. \\
& \left.\left.\quad-\left(c s_{2}+\delta\right)\left(r_{2}-\frac{\omega}{c}-s_{1}\right) e^{s_{2} b}\right)^{-1}\right) e^{s_{1} u}
\end{aligned}
$$$$
V_{2}(u, b)=\frac{\lambda}{\alpha}\left(s_{2}-\frac{\omega}{c}-s_{1}\right) e^{r_{2} u}-\left(r_{2}-\frac{\omega}{c}-s_{1}\right) e^{s_{2} u}
$$$$
\times\left(\left(c r_{2}+\delta\right)\left(s_{2}-\frac{\omega}{c}-s_{1}\right) e^{r_{2} b}\right.
$$$$
\left.-\left(c s_{2}+\delta\right)\left(r_{2}-\frac{\omega}{c}-s_{1}\right) e^{s_{2} b}\right)^{-1} .
$$

2.3. Numerical Examples for $V(u, b)$. As an illustration of the results of the previous subsection, we will give some numerical examples about the expectation of the discounted dividend payments $V(u, b)$.
TABLE 1: Influence of $u$ and $\omega$ on $V(u, b)$ for $\delta=0.1, c=2, \lambda=3$, $\alpha=1$, and $b=10$.

\begin{tabular}{lcccccc}
\hline$u \backslash \omega$ & 1 & 2 & 3 & 4 & 5 & 6 \\
\hline-6 & 1.7969 & 1.2439 & 0.9516 & 0.7707 & 0.6476 & 0.5585 \\
-5 & 1.9620 & 1.3630 & 1.0447 & 0.8471 & 0.7124 & 0.6147 \\
-4 & 2.1422 & 1.4934 & 1.1469 & 0.9311 & 0.7837 & 0.6767 \\
-3 & 2.3390 & 1.6363 & 1.2591 & 1.0234 & 0.8621 & 0.7448 \\
-2 & 2.5539 & 1.7929 & 1.3822 & 1.1249 & 0.9484 & 0.8199 \\
-1 & 2.7885 & 1.9644 & 1.5174 & 1.2364 & 1.0433 & 0.9025 \\
0 & 3.0446 & 2.1524 & 1.6658 & 1.3590 & 1.1478 & 0.9934 \\
1 & 4.4970 & 4.0197 & 3.7594 & 3.5953 & 3.4823 & 3.3997 \\
2 & 5.5086 & 5.2523 & 5.1125 & 5.0243 & 4.9636 & 4.9193 \\
3 & 6.3049 & 6.1662 & 6.0905 & 6.0428 & 6.0010 & 5.9860 \\
4 & 7.0079 & 6.9317 & 6.8901 & 6.8639 & 6.8458 & 6.8326 \\
5 & 7.6841 & 7.6410 & 7.6174 & 7.6026 & 7.5924 & 7.5849 \\
6 & 8.3710 & 8.3453 & 8.3313 & 8.3224 & 8.3163 & 8.3119 \\
7 & 9.0906 & 9.0739 & 9.0649 & 9.0591 & 9.0552 & 9.0523 \\
8 & 9.8568 & 9.8447 & 9.8382 & 9.8340 & 9.8312 & 9.8291 \\
9 & 10.6795 & 10.6697 & 10.6643 & 10.6609 & 10.6586 & 10.6569 \\
\hline
\end{tabular}

Example 2. We set $\delta=0.1, c=2, \lambda=3, \alpha=1$, and $b=10$; it is easy to check that the net profit condition holds; we can discuss impact of the model parameters $u$ and $\omega$ on $V(u, b)$.

Example 3. We set $\delta=0.1, c=2, \lambda=3, \alpha=1$, and $\omega=1$; it is easy to check that the net profit condition holds; we can discuss impact of the model parameters $u$ and $b$ on $V(u, b)$.

Tables 1 and 2 provide numerical results for $V(u, b)$. We find that, for a fixed $\omega$, as can be expected, $V(u, b)$ increases with $u$, The results obtained in Table 1 also illustrate the effect of the bankruptcy parameter $\omega$ for $V(u, b)$. From Table 2, we find that the expectation of the discounted dividend payments $V(u, b)$ decreases with dividend barrier $b$, which makes sense intuitively.

Figure 1 is plotted to illustrate the impact of bankruptcy rate function $\omega(x)$ to dividend payments $V(u, b)$ for various parameter choices $\omega$. Figure 2 is plotted to illustrate the impact of dividend barrier $b$ to dividend payments $V(u, b)$ for various $b$.

\section{The Probability of Bankruptcy $\psi(u, b)$}

\subsection{Integrodifferential Equations for $\psi(u, b)$}

Theorem 4. The probability of bankruptcy $\psi(u, b)$ satisfies the following integrodifferential equations:

$$
\begin{aligned}
& c \psi_{1}^{\prime}(u, b)+(\lambda+\omega(u)) \psi_{1}(u, b)-\omega(u) \\
& -\lambda \int_{u}^{0} \psi_{1}(x, b) f_{X}(x-u) d x-\lambda \int_{0}^{b} \psi_{2}(x, b) \\
& \cdot f_{X}(x-u) d x-\lambda \psi_{2}(b, b) \bar{F}(b-u)=0, \quad u<0,
\end{aligned}
$$




$$
\begin{gathered}
c \psi_{2}^{\prime}(u, b)+\lambda \psi_{2}(u, b)-\lambda \int_{u}^{b} \psi_{2}(x, b) f_{X}(x-u) d x \\
-\lambda \psi_{2}(b, b) \bar{F}(b-u)=0, \quad u \geq 0 .
\end{gathered}
$$

In addition, $\psi_{1}(u, b)$ and $\psi_{2}(u, b)$ satisfy

$$
\begin{gathered}
\psi_{1}\left(0^{-}, b\right)=\psi_{2}\left(0^{+}, b\right), \\
\psi_{2}^{\prime}\left(0^{+}, b\right)-\psi_{1}^{\prime}\left(0^{-}, b\right)=\frac{\omega\left(0^{-}\right)}{c}\left(\psi_{1}\left(0^{-}, b\right)-1\right), \\
\lim _{u \rightarrow-\infty} \psi_{1}(u, b)=1 .
\end{gathered}
$$

Proof. When $u>b$, the surplus drops to level $b$ immediately due to the initial payment of all dividends and thus

$$
\psi_{2}(u, b)=\psi_{2}(b, b), \quad u>b .
$$

When $u<0$, by conditioning on the first occurrence time and amount of the gain or an event of bankruptcy up to time $t$,

$$
\begin{aligned}
\psi_{1}(u, b)= & (1-\lambda t)(1-\omega(u) t) \psi_{1}(u-c t, b) \\
& +(1-\lambda t) \omega(u) t+\lambda t(1-\omega(u) t) \\
& \cdot\left[\int_{0}^{-u+c t} \psi_{1}(u-c t+x, b) f_{X}(x) d x\right. \\
& +\int_{-u+c t}^{b-u+c t} \psi_{2}(u-c t+x, b) f_{X}(x) d x \\
& \left.+\int_{b-u+c t}^{\infty} \psi_{2}(b, b) f_{X}(x) d x\right] \\
+ & o(t)=0 .
\end{aligned}
$$

Differentiating (39) with respect to $t$ and taking the limit $t \rightarrow$ 0 we can obtain (33).

Using a similar argument, we can also derive the corresponding bankruptcy probability $\psi_{2}(u, b)$ for $0 \leq u<b$. The conditions (35)-(37) are also obvious.

3.2. Explicit Expressions for $\psi(u, b)$. In this subsection, we assume that $\omega(u)$ is also constant $\omega$, and the gain size follows $f(x)=\alpha e^{-\alpha x}$; then (33) and (34) can be rewritten as

$$
\begin{aligned}
& c \psi_{1}^{\prime}(u, b)+(\lambda+\omega) \psi_{1}(u, b)-\omega \\
& -\lambda \int_{u}^{0} \psi_{1}(x, b) \alpha e^{-\alpha x} e^{\alpha u} d x \\
& -\lambda \int_{0}^{b} \psi_{2}(x, b) \alpha e^{-\alpha x} e^{\alpha u} d x \\
& -\lambda \psi_{2}(b, b) e^{-\alpha(b-u)}=0, \\
& c \psi_{2}^{\prime}(u, b)+\lambda \psi_{2}(u, b)-\lambda \int_{u}^{b} \psi_{2}(x, b) \alpha e^{-\alpha x} e^{\alpha u} d x \\
& -\lambda \psi_{2}(b, b) e^{-\alpha(b-u)}=0 .
\end{aligned}
$$

TABLE 2: Influence of $u$ and $b$ on $V(u, b)$ for $\delta=0.1, c=2, \lambda=3$, $\alpha=1$, and $\omega=1$.

\begin{tabular}{lcccccc}
\hline$u \backslash b$ & 10 & 11 & 12 & 13 & 14 & 15 \\
\hline-6 & 1.7969 & 1.6610 & 1.5348 & 1.4179 & 1.3097 & 1.2098 \\
-5 & 1.9620 & 1.8136 & 1.6758 & 1.5481 & 1.4300 & 1.3209 \\
-4 & 2.1422 & 1.9802 & 1.8297 & 1.6903 & 1.5614 & 1.4422 \\
-3 & 2.3390 & 2.1621 & 1.9978 & 1.8456 & 1.7048 & 1.5747 \\
-2 & 2.5539 & 2.3607 & 2.1813 & 2.0151 & 1.8614 & 1.7194 \\
-1 & 2.7885 & 2.5776 & 2.3817 & 2.2002 & 2.0324 & 1.8773 \\
0 & 3.0446 & 2.8144 & 2.6005 & 2.40230 & 2.2191 & 2.0497 \\
1 & 4.4970 & 4.1569 & 3.8409 & 3.5483 & 3.2777 & 3.0275 \\
2 & 5.5086 & 5.0919 & 4.7049 & 4.3465 & 4.0150 & 3.7085 \\
3 & 6.3049 & 5.8281 & 5.3851 & 4.9749 & 4.5954 & 4.2447 \\
4 & 7.0079 & 6.4779 & 5.9855 & 5.5295 & 5.1078 & 4.7179 \\
5 & 7.6841 & 7.1029 & 6.5631 & 6.0631 & 5.6006 & 5.1732 \\
6 & 8.3710 & 7.7379 & 7.1498 & 6.6051 & 6.1013 & 5.6356 \\
7 & 9.0906 & 8.4030 & 7.7644 & 7.1728 & 6.6257 & 6.1201 \\
8 & 9.8568 & 9.1113 & 8.4188 & 7.77740 & 7.1842 & 6.6359 \\
9 & 10.6795 & 9.8718 & 9.1215 & 8.4266 & 7.7839 & 7.1898 \\
\hline
\end{tabular}

Applying the operators $(d / d x-\alpha)$ to $(40)$, they can be rewritten as

$$
\begin{gathered}
c \psi_{1}^{\prime \prime}(u, b)+(\lambda+\omega-c \alpha) \psi_{1}^{\prime}(u, b)-\omega \alpha \psi_{1}(u, b)+\omega \alpha=0 . \\
c \psi_{2}^{\prime \prime}(u, b)+(\lambda-c \alpha) \psi_{2}^{\prime}(u, b)=0 .
\end{gathered}
$$

We know the solution of (41) is of the form

$$
\psi_{1}(u, b)=1+A_{1}^{\prime} e^{-r_{1}^{\prime} u}+B_{1}^{\prime} e^{s_{1}^{\prime} u}
$$

where $A_{1}^{\prime}, B_{1}^{\prime}$ are constants and $-r_{1}^{\prime}<0, s_{1}^{\prime}>0$ are the solutions of the equation about $R$ :

$$
c R^{2}+(\lambda+\omega-c \alpha) R-\omega \alpha=0 .
$$

From $\lim _{u \rightarrow-\infty} \psi_{1}(u, b)=1, A_{1}^{\prime}=0$ is obvious.

For $u \geq 0$, we obtain the solution of (42) which is of the form

$$
\psi_{2}(u, b)=A_{1}^{\prime \prime} e^{(\alpha-(\lambda / c)) u}+B_{1}^{\prime \prime} .
$$

When $u \rightarrow \infty$, we have $\psi_{2}(u, b) \rightarrow 0$. As $\lambda / \alpha-c>0$, that is, $\alpha-\lambda / \alpha<0$, so $B_{1}^{\prime \prime}=0$ is obvious. Then

$$
\psi_{2}(u, b)=A_{1}^{\prime \prime} e^{(\alpha-\lambda / \alpha) u} .
$$

Similar to deriving $V(u, b)$, we have that the conditions (35) and (36) for $\psi(u, b)$ give that

$$
1+B_{1}^{\prime}=A_{1}^{\prime \prime}, \quad A_{1}^{\prime \prime}\left(\alpha-\frac{\lambda}{c}\right)-B_{1}^{\prime} s_{1}^{\prime}=\frac{\omega}{c} B_{1}^{\prime} .
$$

We have

$$
A_{1}^{\prime \prime}=\frac{c s_{1}^{\prime}+\omega}{c s_{1}^{\prime}+\omega-c \alpha+\lambda}, \quad B_{1}^{\prime}=\frac{c \alpha-\lambda}{c s_{1}^{\prime}+\omega-c \alpha+\lambda} .
$$




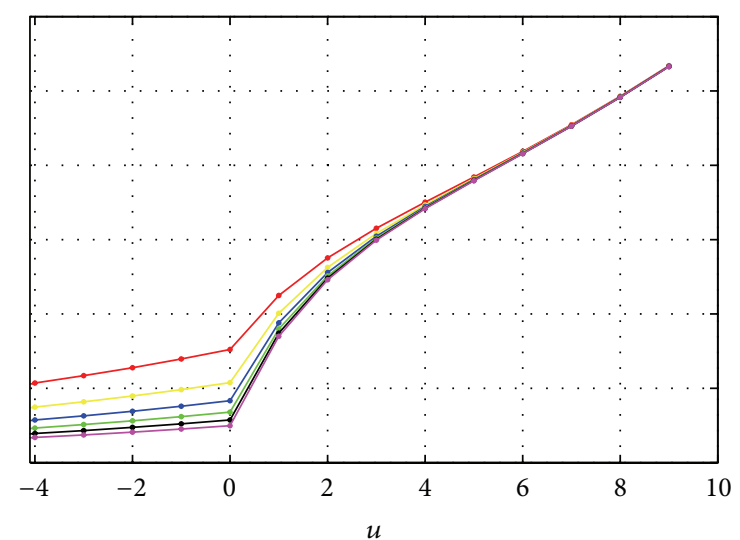

$\begin{array}{ll}\text { Red: } \omega=1 & \text { Green: } \omega=4 \\ \text { Yellow: } \omega=2 & \text { Black: } \omega=5 \\ \text { Blue: } \omega=3 & \text { Purple: } \omega=6\end{array}$

FIGURE 1: The expected sum of discounted dividend $V(u, b)$ when $b=10$.

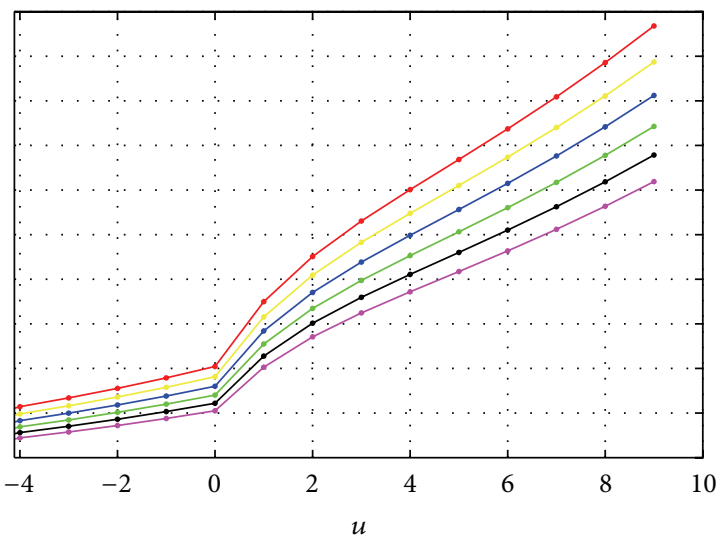

$$
\begin{array}{ll}
\text { Red: } b=10 & \text { Green: } b=13 \\
\text { Yellow: } b=11 & \text { Black: } b=14 \\
\text { Blue: } b=12 & \text { Purple: } b=15
\end{array}
$$

FIgURE 2: The expected sum of discounted dividend $V(u, b)$ when $\omega=1$.

So

$$
\begin{aligned}
& \psi_{1}(u, b)=1+\frac{c \alpha-\lambda}{c s_{1}^{\prime}+\omega-c \alpha+\lambda} e^{s_{1}^{\prime} u}, \\
& \psi_{2}(u, b)=\frac{c s_{1}^{\prime}+\omega}{c s_{1}^{\prime}+\omega-c \alpha+\lambda} e^{(\alpha-\lambda / \alpha) u} .
\end{aligned}
$$

Example 5. We can perform analysis for the probability of bankruptcy $\psi(u, b)$; again we choose $\delta=0.1, c=2, \lambda=3, \alpha=$ 1 , and $b=10$.

In Table 3 we find that, for a given $u$, the probability of bankruptcy $\psi(u, b)$ increases with $u$. And it also can be expected, as a large value of bankruptcy rate $\omega$ implies the large bankruptcy probability $\psi(u, b)$.
TABLE 3: Influence of $u$ and $\omega$ on $\psi(u, b)$ for $\delta=0.1, c=2, \lambda=3$, $\alpha=1$, and $b=10$.

\begin{tabular}{lcccccc}
\hline$u \backslash \omega$ & 1 & 2 & 3 & 4 & 5 & 6 \\
\hline-6 & 0.9593 & 0.9876 & 0.9941 & 0.9965 & 0.9977 & 0.9983 \\
-5 & 0.9413 & 0.9795 & 0.9894 & 0.9934 & 0.9955 & 0.9966 \\
-4 & 0.9153 & 0.9662 & 0.9811 & 0.9876 & 0.9910 & 0.9931 \\
-3 & 0.8779 & 0.9442 & 0.9661 & 0.9765 & 0.9823 & 0.9860 \\
-2 & 0.8240 & 0.9080 & 0.9394 & 0.9555 & 0.9651 & 0.9714 \\
-1 & 0.7462 & 0.8484 & 0.8917 & 0.9158 & 0.9311 & 0.9418 \\
0 & 0.6340 & 0.7500 & 0.8063 & 0.8406 & 0.8641 & 0.8813 \\
1 & 0.3845 & 0.4549 & 0.4890 & 0.5099 & 0.5241 & 0.5345 \\
2 & 0.2332 & 0.2759 & 0.2966 & 0.3092 & 0.3179 & 0.3242 \\
3 & 0.1415 & 0.1673 & 0.1799 & 0.1876 & 0.1928 & 0.1966 \\
4 & 0.0858 & 0.1015 & 0.1091 & 0.1138 & 0.1169 & 0.1193 \\
5 & 0.0520 & 0.0616 & 0.0662 & 0.0690 & 0.0709 & 0.0723 \\
6 & 0.0316 & 0.0373 & 0.0401 & 0.0418 & 0.0430 & 0.0439 \\
7 & 0.0191 & 0.0226 & 0.0243 & 0.0254 & 0.0261 & 0.0266 \\
8 & 0.0116 & 0.0137 & 0.0148 & 0.0154 & 0.0158 & 0.0161 \\
9 & 0.0070 & 0.0083 & 0.0090 & 0.0093 & 0.0096 & 0.0098 \\
\hline
\end{tabular}

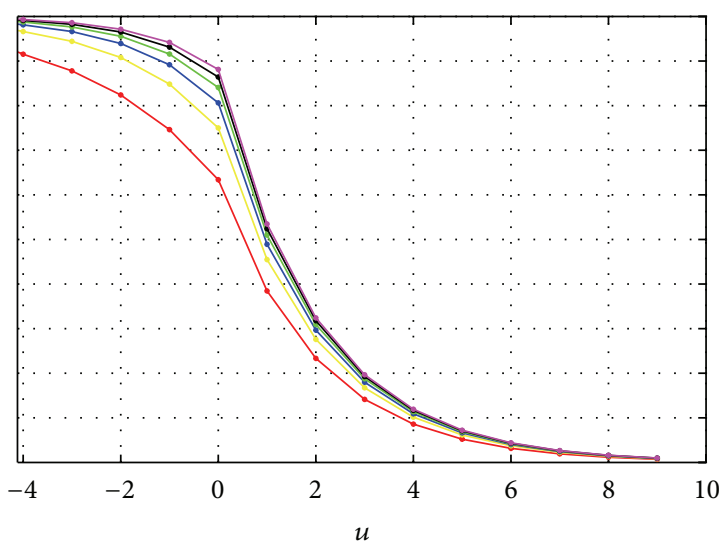

$\begin{array}{ll}\text { Red: } \omega=1 & \text { Green: } \omega=4 \\ \text { Yellow: } \omega=2 & \text { Black: } \omega=5 \\ \text { Blue: } \omega=3 & \text { Purple: } \omega=6\end{array}$

FIGURE 3: The bankruptcy probability $\psi(u, b)$ when $b=10$.

Figure 3 was produced by the explicit expression in (49); it is plotted to illustrate the impact of $\omega$ and $u$ on bankruptcy probability $\psi(u, b)$.

\section{Conflict of Interests}

The authors declare that there is no conflict of interests regarding the publication of this paper.

\section{Acknowledgments}

This research is fully supported by a Grant from Natural Science Foundation of Hunan (13JJ4083), by Humanities and Social Sciences Project of the Ministry Education in China (10YJC630144), by Hunan Social Science Fund Program 
(12YBB093), and by Scientific Fund of Hunan Provincial Education Department (13C318) and is also supported by Natural Science Foundation of Anhui Higher Education Institutions (KJ2014ZD21).

\section{References}

[1] B. De Finetti, "Su un'impostazione alternativa della teoria collettiva del rischio," in Proceedings of the Transactions of the 15th International Congress of Actuaries, vol. 2, pp. 433-443, 1957.

[2] B. Avanzi, H. U. Gerber, and E. S.W. Shiu, "Optimal dividends in the dual model," Insurance: Mathematics and Economics, vol. 41, no. 1, pp. 111-123, 2007.

[3] B. Avanzi and H. U. Gerber, "Optimal dividends in the dual model with diffusion," ASTIN Bulletin, vol. 38, no. 2, pp. 653667, 2008.

[4] A. C. Y. Ng, "On a dual model with a dividend threshold," Insurance: Mathematics \& Economics, vol. 44, no. 2, pp. 315-324, 2009.

[5] H. Dai, Z. Liu, and N. Luan, "Optimal dividend strategies in a dual model with capital injections," Mathematical Methods of Operations Research, vol. 72, no. 1, pp. 129-143, 2010.

[6] H. Albrecher and V. Lautscham, "From ruin to bankruptcy for compound poisson surplus processes," ASTIN Bulletin, vol. 43, no. 2, pp. 213-243, 2013. 


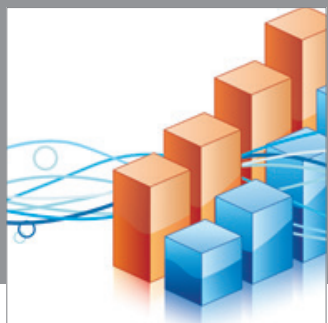

Advances in

Operations Research

mansans

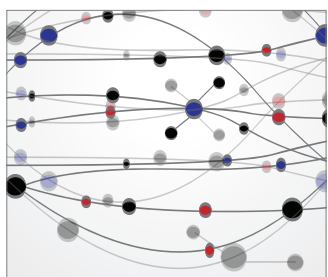

The Scientific World Journal
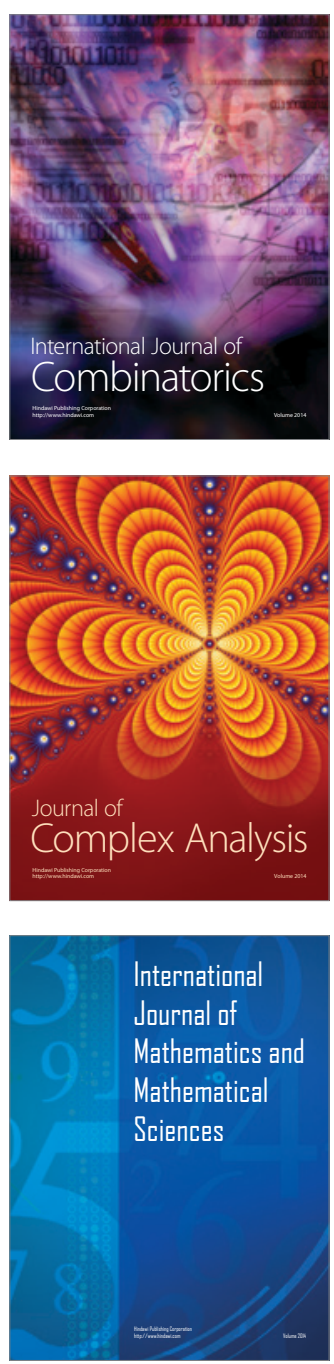
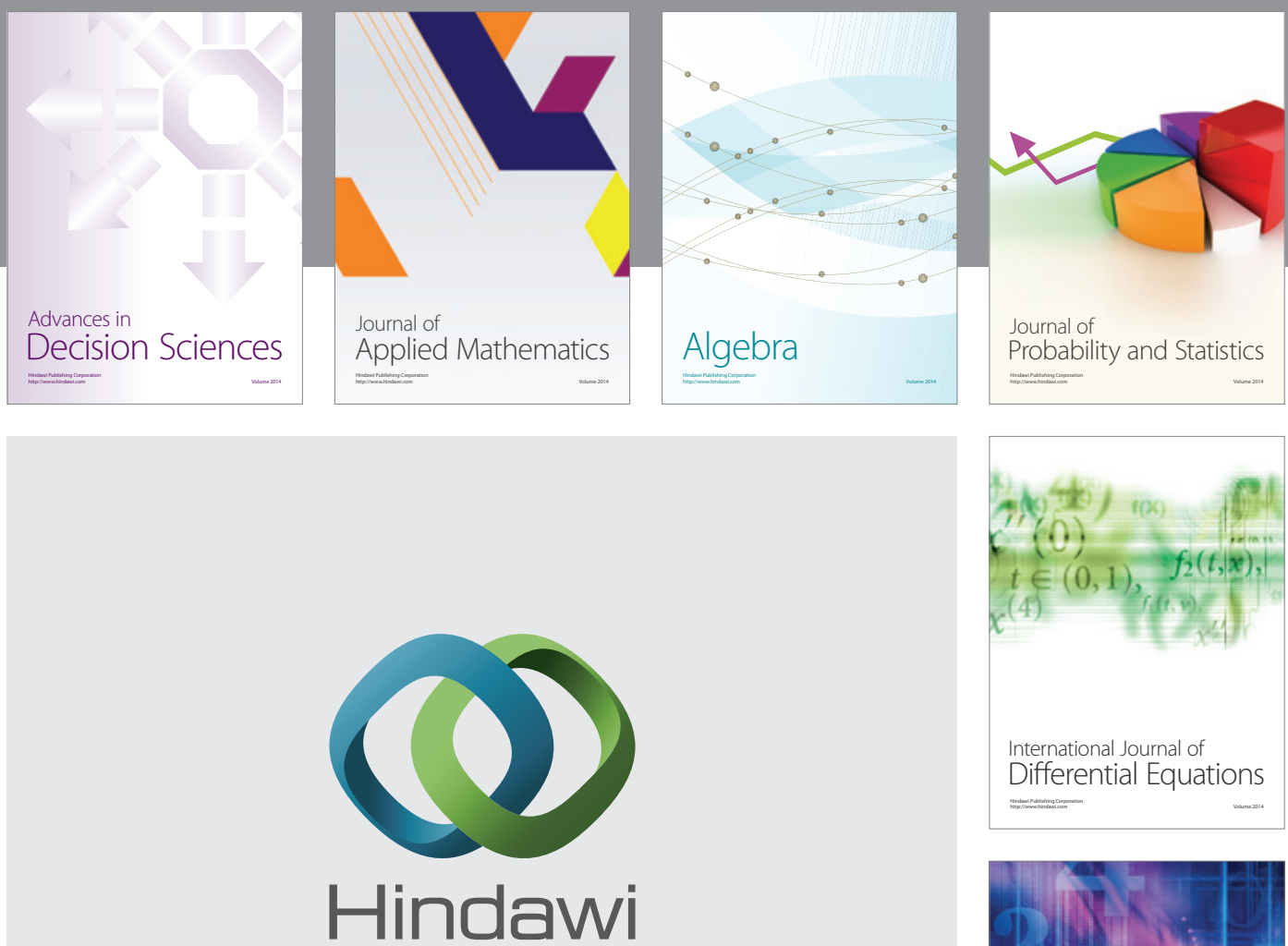

Submit your manuscripts at http://www.hindawi.com
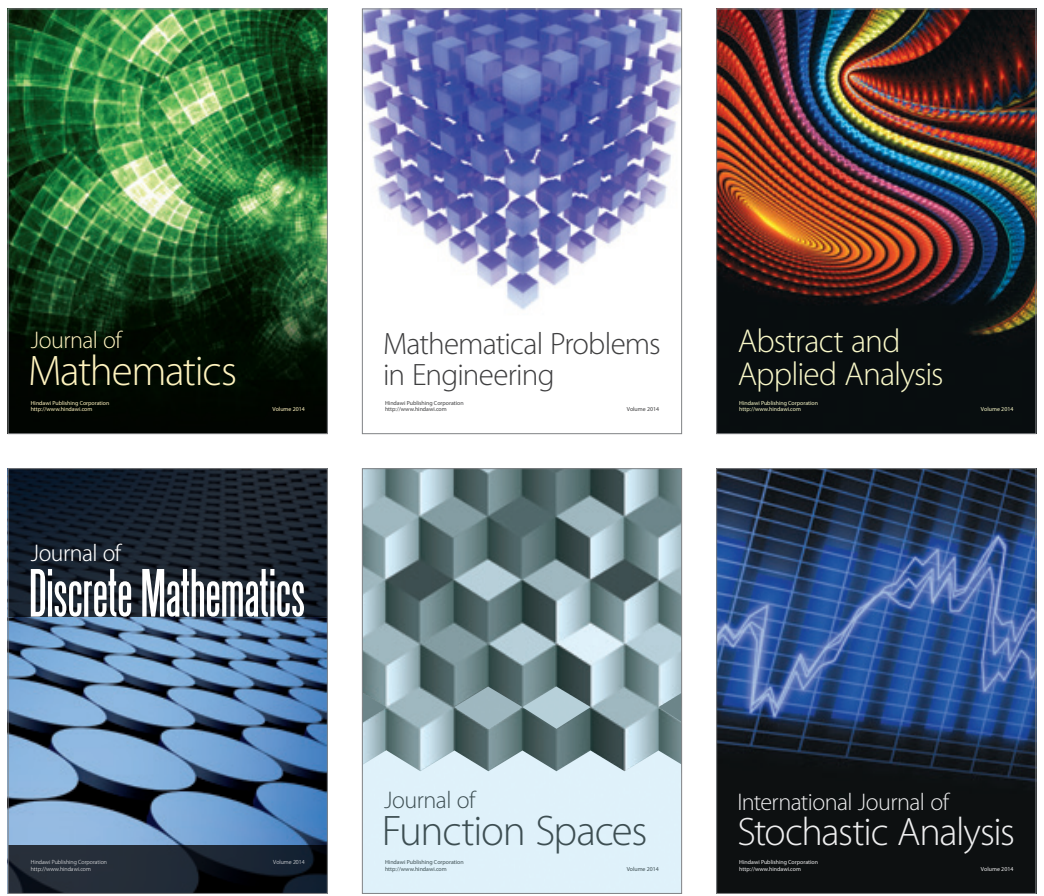

Journal of

Function Spaces

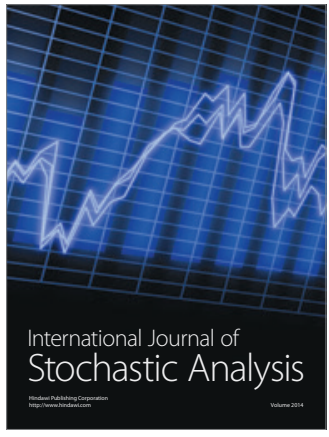

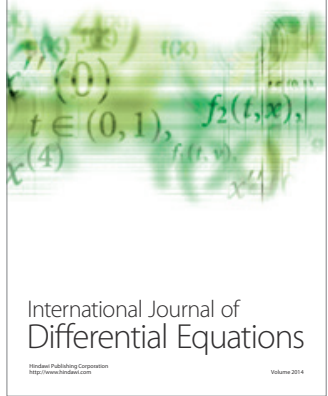
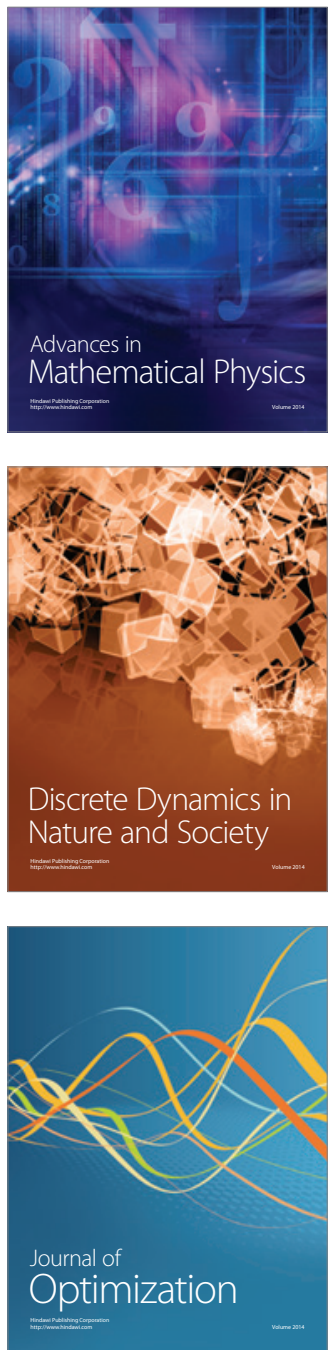